\title{
Hiperparatiroidismo primario
}

\author{
Primary hyperparathyroidism
}

Carlos E. Builes-Montaño MD'

Resumen: el hiperparatiroidismo primario es uno de los trastornos endocrinos más frecuentes, caracterizado por la producción no controlada de la paratohormona. Esta condición clínica puede ser completamente asintomática o mostrarse con manifestaciones que ponen en peligro la vida de quien la padece. La gran variedad en las formas de presentación y algunos errores frecuentes en la interpretación de los resultados de las pruebas disponibles para su diagnóstico hacen de esta alteración un reto para el médico. Un hiperparatiroidismo no diagnosticado, y en consecuencia no tratado, puede tener consecuencias nefastas para los pacientes como deterioro en la función renal, que puede llevar a requerir una terapia de reemplazo renal, osteoporosis, que se puede acompañar de fracturas por fragilidad y cambios neurocognitivos que pueden socavar la calidad de vida de las personas. Por lo anterior, es fundamental conocer y comprender este trastorno, así como reconocer e interpretar adecuadamente las ayudas diagnósticas para poder proponer un plan de tratamiento adecuado que, afortunadamente en la mayoría de los casos, lleva a la curación del trastorno.

Palabras clave: hiperparatiroidismo primario, hormona paratiroidea, hipercalcemia.

Builes-Montaño CE. Hiperparatiroidismo primario. Medicina \& Laboratorio 2017; 23: 45-64.

$\mathbf{E}$ hiperparatiroidismo primario es un desorden endocrino, caracterizado por una producción no controlada de la paratohormona (PTH) y que se puede acompañar, o no, de una elevación en los niveles de calcio circulantes, un aumento en la excreción renal de calcio, enfermedad ósea, enfermedad renal y toda una constelación de síntomas y signos clínicos. El hiperparatiroidismo primario puede ser consecuencia de la presencia de un adenoma en una de las glándulas paratiroides o de una hiperplasia de todas ellas. Se puede manifestar como una condición aislada o como parte de un síndrome clínico hereditario, acompañando otras alteraciones endocrinas $y$ no endocrinas [1]. En este manuscrito se revisan exclusivamente las características principales del hiperparatiroidismo primario con énfasis en la forma más común de presentación que es la producida por un adenoma paratiroideo.

${ }^{1}$ Médico, especialista en Medicina Interna y Endocrinología Clínica y Metabolismo. Endocrinólogo, Hospital Pablo Tobón Uribe. Profesor auxiliar, Universidad de Antioquia. Medellín,

Colombia.Correo electrónico: cbuiles@hptu.org.co

Conflicto de intereses: el autor declara que no tiene conflicto de intereses Medicina \& Laboratorio 2017; 23: 45-64

Módulo 2 (Endocrinología), número 16. Editora Médica Colombiana S.A. 2017@)

Recibido el 04 de febrero de 2017; aceptado el 27 de febrero de 2017 


\section{Epidemiología}

A principios del siglo $x x$ se consideraba al hiperparatiroidismo primario como un trastorno exótico debido a que su forma de presentación "clásica», la osteítis fibrosa quística, era una condición sumamente infrecuente. En 1934 Fuller Albright y colaboradores cambian este concepto al sugerir que el hiperparatiroidismo primario es una condición frecuente y que puede tener diversas formas de presentación clínica [2].

Hasta la segunda mitad del siglo xx no era posible medir de manera sencilla y rápida los niveles de calcio en la sangre, pero esto cambió con el uso rutinario de analizadores multicanal alrededor del año 1970. Debido a esto, era usual que en la gran mayoría de los casos el hiperparatiroidismo primario se detectara solo cuando presentaba alguna de sus formas sintomáticas típicas como consecuencia de los altos niveles de calcio en la sangre, el compromiso esquelético o renal [3].

Hoy día es mucho más frecuente encontrar personas con niveles de calcio en sangre elevados y un valor de paratohormona elevado o inapropiadamente normal, pero sin ninguna manifestación clínica [3]. De hecho, la proporción de pacientes adultos sintomáticos viene disminuyendo en todo el mundo, como lo demuestran varios reportes en los que se registra que para el año 1961 un 95\% de los casos de hiperparatiroidismo primario eran sintomáticos mientras que para el año 2013 esta proporción disminuyó a valores por debajo del $20 \%[4,5]$.

Actualmente, el hiperparatiroidismo primario es una alteración endocrina relativamente frecuente, con prevalencias reportadas entre el $0,1 \%$ hasta el $0,8 \%$ de la población adulta, donde alrededor del $80 \%$ de los casos son asintomáticos. Este trastorno se presenta con mayor frecuencia en las mujeres posmenopáusicas con una proporción mujer hombre de 3-7:1. Además, el hiperparatiroidismo primario es la principal causa de hipercalcemia en las personas que no se encuentran hospitalizadas $[5,6]$.

Un reporte de la población de Rochester, Minnesota, Estados Unidos, permitió apreciar cómo la incidencia de la enfermedad varía en función del tiempo de acuerdo a diferentes factores políticos, sociales y técnicos. Por ejemplo, en la década del setenta, cuando se hizo posible la medición rutinaria de calcio, se presentó un marcado incremento en la incidencia con una posterior disminución, lo cual se atribuye al efecto de barrido ocasionado por el diagnóstico de las personas previamente "sanas» que antes no habían podido ser detectadas. En la década del ochenta se presenta nuevamente un pequeño incremento en la incidencia, lo que logra, probablemente, alcanzar la incidencia esperada de la enfermedad. En la década del noventa los autores refieren que se dejan de hacer mediciones rutinarias de calcio, lo que provoca una nueva disminución en la incidencia, pero nunca hasta lograr los niveles iniciales en los que la mayoría de las personas presentaban síntomas de hiperparatiroidismo primario (véase figura 1) [7].

Es importante resaltar que existen diferencias en la forma de presentación del hiperparatiroidismo primario en relación con la edad. En las personas jóvenes, alrededor de los 25 y 30 años, la presencia de hipercalcemia con niveles inapropiados de paratohormona debe sugerir que la causa del hiperparatiroidismo primario es un síndrome familiar, siendo el más común la neoplasia endocrina múltiple tipo 1 , la cual tiene una alta frecuencia de presentación con hiperparatiroidismo primario antes de los 30 años y tiende a presentarse como una enfermedad 


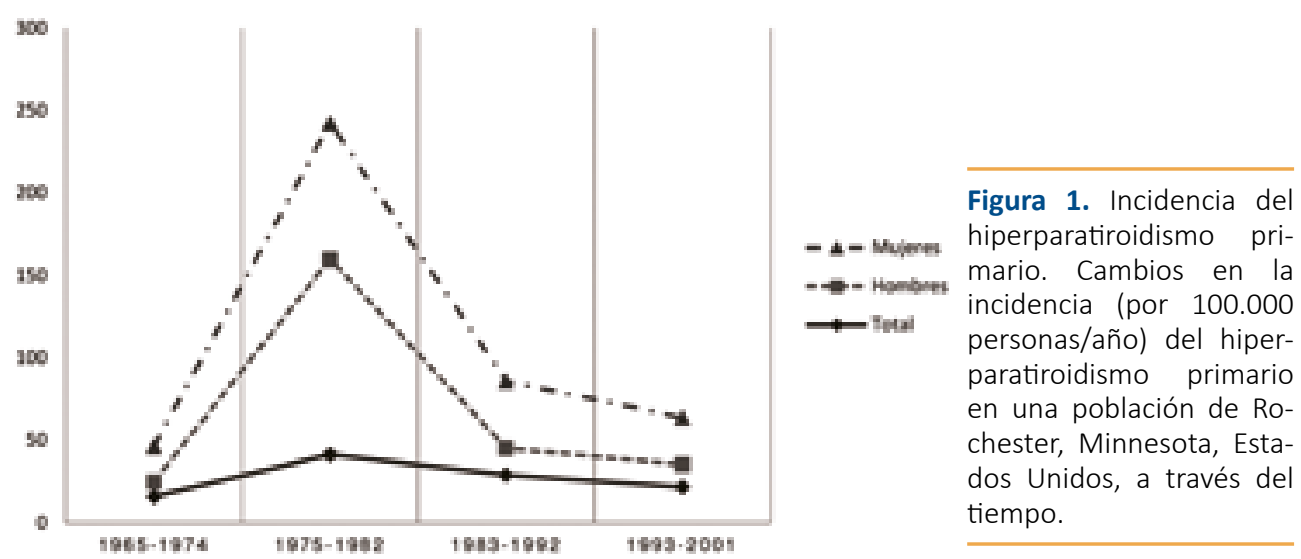

poliglandular [8]. Además, el hiperparatiroidismo primario en las personas jóvenes tiende a presentarse con valores de calcio mayores, a pesar de no haber diferencias en el valor de la paratohormona en comparación con los individuos más adultos; esto se podría explicar por la mayor incidencia de la enfermedad poliglandular o, de acuerdo a algunos autores, podría constituir un artefacto estadístico debido al uso rutinario de la medición de calcio en los adultos [9].

Algunos factores se han reportado asociados a un aumento en el riesgo de presentar hiperparatiroidismo primario o que modifican el metabolismo del calcio, lo que dificulta la interpretación de las mediciones de la paratohormona. Por ejemplo, la exposición a radiación ionizante en el cuello se ha asociado con un incremento en la incidencia del hiperparatiroidismo primario de 2,5 veces luego de tres o cuatro décadas de la exposición [10]. El consumo de diuréticos tiazídicos es una causa reconocida de hipercalcemia, cuyas características clínicas se asemejan a las del hiperparatiroidismo primario y usualmente se presenta en mujeres después de la sexta década de la vida; una vez se suspende el medicamento la hipercalcemia persiste en una proporción importante de los pacientes, lo que sugiere que el uso de este grupo de medicamentos puede enmascarar un hiperparatiroidismo primario al atribuírsele la hipercalcemia [11]. El litio es otro medicamento implicado en el hiperparatiroidismo primario, el cual usualmente, después de su inicio, induce de forma rápida una ligera elevación en los niveles de calcio debido a un cambio en el umbral de estimulación mediado por el calcio en las células paratiroideas; en la mayoría de los casos el calcio retorna al nivel normal una vez se suspende el medicamento, pero con el uso crónico del mismo alrededor del $10 \%$ de los pacientes pueden desarrollar un hiperparatiroidismo primario causado por una hiperplasia o un adenoma paratiroideo [12].

\section{Manifestaciones y formas clínicas}

La forma de presentación del hiperparatiroidismo primario ha cambiado a través del tiempo y, como se mencionó previamente, esto probablemente es consecuencia de la facilidad y la frecuencia con la cual se hace la medición de calcio, como lo han demostrado algunos reportes de países donde con la introducción de analizadores multicanal 
la proporción de pacientes diagnosticados en ausencia de síntomas viene en aumento [13].

De manera práctica podemos agrupar las diferentes formas de presentación del hiperparatiroidismo primario según los sistemas comprometidos. En un mismo paciente se pueden presentar varios de los síntomas de la enfermedad. Algunos han sido relacionados principalmente con la hipercalcemia en la forma sintomática del hiperparatiroidismo primario, entre los que se incluyen la úlcera péptica, la pancreatitis, las náuseas, el vómito, la constipación, la pérdida de peso, la polidipsia y la alteración del estado de conciencia.

\section{Hiperparatiroidismo primario asintomático}

Usualmente la sospecha de un hiperparatiroidismo primario asintomático comienza con el hallazgo incidental de un calcio sérico elevado en un paciente sin síntomas clásicos. Debido a que no es inusual que un nivel elevado de calcio sea un error analítico, siempre es necesario repetir su medición antes de solicitar estudios adicionales. En el contexto de un calcio elevado, un valor de la paratohormona elevado o normal debe hacer sospecha de un hiperparatiroidismo primario; de hecho, los valores inapropiadamente normales son frecuentes, presentándose hasta en la mitad de los pacientes con hiperparatiroidismo primario [14]. Esta condición, en la que los niveles de calcio se encuentran elevados pero la paratohormona no supera el valor de referencia, se conoce como hiperparatiroidismo primario no clásico, en el cual la falta de supresión en la producción de la paratohormona es indicativa de un defecto en el mecanismo de retroalimentación negativa. Esta forma de hiperparatiroidismo primario es fuente frecuente de confusión y produce retrasos en el diagnóstico y un seguimiento inadecuado [14].

En la tabla 1 se resumen las recomendaciones actuales para la evaluación y el manejo del hiperparatiroidismo primario asintomático [15]. Aproximadamente el 55\% de los pacientes con un hiperparatiroidismo primario no clásico son diagnosticados en el seguimiento [16]; además, tienen seis veces más riesgo de presentar una fractura vertebral [17] y cuatro veces más riesgo de ser diagnosticados con cálculos renales [18].

\section{Manifestaciones óseas del hiperparatiroidismo}

De acuerdo a los reportes de diferentes áreas geográficas las manifestaciones óseas del hiperparatiroidismo primario se encuentran cada vez con menos frecuencia. La manifestación esquelética más severa del hiperparatiroidismo primario es la osteítis fibrosa quística, una condición supremamente infrecuente. Otras más frecuentes incluyen el dolor óseo, la baja densidad mineral y las fracturas por fragilidad. Es de anotar que estos pacientes usualmente presentan otras manifestaciones de la enfermedad como síntomas neuropsiquiátricos, manifestaciones renales y debilidad muscular [1].

Las manifestaciones óseas se caracterizan generalmente por lesiones en «sal y pimienta» en el cráneo, erosión y resorción en las falanges, tumores pardos y quistes. Además, se puede acompañar de una densidad mineral ósea baja, usualmente más disminuida cuando se compara con aquella que se presenta sin hiperparatiroidismo primario en otras formas de osteoporosis. Generalmente, se afectan con mayor frecuencia los huesos con un alto contenido de hueso cortical debido al efecto metabólico de la 
Tabla 1. Guías para el seguimiento y tratamiento del hiperparatiroidismo primario asintomático

\begin{tabular}{ll}
\hline Guías para cirugía en hiperparatiroidismo primario asintomático \\
\hline Criterio & Indicación \\
Calcio sérico medido & $\begin{array}{l}1,0 \mathrm{mg} / \mathrm{dL} \text { por encima del valor de referencia del laboratorio } \\
\text { Esquelético }\end{array}$ \\
$\begin{array}{l}\text { Densidad mineral ósea por absorciometría de rayos } \mathrm{X} \text { de energía dual: puntuación T } \\
\text { menor que-2,5 en columna lumbar, cadera, cuello femoral o tercio distal del radio }\end{array}$ \\
$\begin{array}{l}\text { Fracturas vertebrales detectadas por rayos } \mathrm{X} \text {, tomografía, resonancia magnética o } \\
\text { morfometría }\end{array}$
\end{tabular}

Renal

Depuración de creatinina inferior a $60 \mathrm{~mL} / \mathrm{min}$

Calcio en orina de 24 horas superior a $400 \mathrm{mg} /$ día, con un riesgo alto para formación de cálculos en el análisis bioquímico de riesgo para cálculos ${ }^{b}$

Nefrolitiasis o nefrocalcinosis diagnosticada por rayos $X$, ultrasonido o tomografía

Edad (años)

$<50$ años

a. En mujeres premenopaúsicas y en hombres menores de 50 años se debe utilizar el puntaje Z.

b. El análisis bioquímico de riesgo para cálculos está compuesto de la medición en orina de 24 horas de calcio, citrato, oxalato, creatinina y ácido úrico.

Nota: La cirugía también está indicada en aquellos pacientes en los que no se pueda hacer un seguimiento adecuado o cuando el paciente lo desee, siempre y cuando no tenga contraindicaciones.

\begin{tabular}{|c|c|}
\hline \multicolumn{2}{|c|}{ Guías para el seguimiento en hiperparatiroidismo primario asintomático } \\
\hline Criterio & Indicación \\
\hline Calcio sérico & Medición anual \\
\hline \multirow[t]{2}{*}{ Esquelético } & $\begin{array}{l}\text { Densidad mineral ósea por absorciometría de rayos } X \text { de energía dual cada uno a dos } \\
\text { años }\end{array}$ \\
\hline & $\begin{array}{l}\text { Rayos X o morfometría vertebral si hay criterio clínico (p. ej. disminución de estatura, } \\
\text { dolor dorsal o lumbar) }\end{array}$ \\
\hline \multirow[t]{2}{*}{ Renal } & Creatinina sérica anual \\
\hline & $\begin{array}{l}\text { Si se sospecha urolitiasis: ultrasonografía o tomografía renal, análisis bioquímico de } \\
\text { riesgo para cálculos }\end{array}$ \\
\hline
\end{tabular}

Nota: si el paciente desarrolla durante el seguimiento alguno de los criterios mencionados previamente para cirugía se le debe ofrecer esta opción de tratamiento

paratohormona sobre el osteoclasto, lo que aumenta su profundidad de resorción en la superficie endocortical; esta baja masa ósea tiende a mejorar de manera significativa después del tratamiento [19].

La frecuencia con la cual se reportan estos hallazgos es altamente variable dependiendo de la población estudiada y el momento histórico en el cual se hace el reporte. Por ejemplo, en la descripción inicial hecha por Albright y colaboradores (1934) el 70\% de los pacientes diagnosticados en un hospital de Estados Unidos tenía algún grado de compromiso óseo [2], mientras que en un reporte reciente (2013), donde se revisaron más de 4.000 casos de hiperparatiroidismo primario en Brasil, las manifestaciones óseas fueron infrecuentes, encontrándose en alrededor del $6 \%$ de los pacientes con hiperparatiroidismo primario [5].

En los rayos $X$ de los pacientes con manifestaciones esqueléticas es frecuente encontrar una desmineralización difusa, y en aquellos con osteítis fibrosa quística erosiones subperiósticas de las falanges distales o medias (véase figura 2), desaparición radiológica 


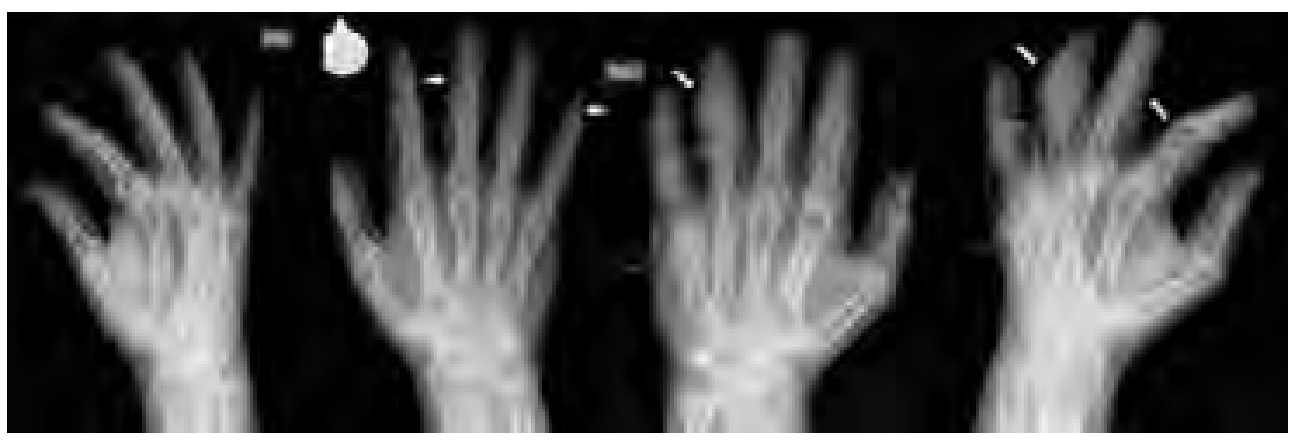

Figura 2. Manifestaciones radiológicas de la osteítis fibrosa quística. Las flechas señalan erosiones subperiósticas en una falange distal y en las porciones laterales de las falanges medias; además, un tumor pardo en dos de las falanges medias.

del tercio distal de la clavícula, lesiones de apariencia quísticas en los huesos, lesiones en «sal y pimienta» en el cráneo y tumores pardos, generalmente acompañados de dolor óseo [20].

Los marcadores de recambio óseo reflejan la función celular osteoblástica (osteocalcina, propéptido $\mathrm{N}$-terminal del procolágeno 1 y la isoforma de la fosfatasa alcalina específica del hueso) y osteoclástica (telopéptidos C- y $\mathrm{N}$-terminal del colágeno tipo 1 , deoxipiridolina y la fosfatasa acida resistente al tartrato). Una proporción importante de pacientes con hiperparatiroidismo primario tienen elevación de los marcadores de recambio que se normalizan con la cirugía, la terapia con estrógenos o con antiresortivos; sin embargo, no se puede hacer una recomendación para su uso de manera rutinaria debido a la variabilidad biológica y tecnológica [21].

\section{Manifestaciones renales}

El riñón es probablemente el órgano más frecuentemente afectado en el hiperparatiroidismo primario, en el cual se puede producir nefrolitiasis y nefrocalcinosis. Algunos reportes muestran cómo hasta la mitad de los pacientes afectados tiene compromiso renal [13].
El riñón desempeña un papel fundamental en la regulación de los niveles sanguíneos de calcio y fósforo mediante la filtración, reabsorción y excreción. La paratohormona regula la reabsorción renal de calcio y fósforo, lo que hace que en el hiperparatiroidismo primario se altere la relación filtración/ excreción de calcio y, en consecuencia, que incremente la reabsorción. La paratohormona disminuye la reabsorción renal de fósforo, lo que disminuye la actividad del cotransportador $\mathrm{Na} / \mathrm{Pi}$ y, por lo tanto, favorece la excreción de fósforo. Además, la paratohormona regula la actividad de la enzima $1 \alpha$-hidroxilasa renal para incrementar la concentración de la 1,25-dihidroxi vitamina $D$, lo que aumenta la absorción intestinal de calcio. Así pues, se configura el cuadro bioquímico clásico del hiperparatiroidismo primario: niveles elevados de la paratohormona, altos niveles de calcio y bajos de fósforo en suero, altos niveles séricos de 1,25-dihidroxi vitamina $D$ e hipercalciuria.

La causa de la urolitiasis en el hiperparatiroidismo primario es multifactorial ya que en este fenómeno converge el aumento en la concentración urinaria de calcio, fósforo y oxalato, además de un incremento en el 
$\mathrm{pH}$ urinario. Todos estos factores son reconocidos en la formación de cálculos renales; no obstante, y a pesar de ser una de las manifestaciones clínicas más comunes, solo una pequeña proporción de pacientes los presenta [22], lo que hace pensar que otros factores deben estar implicados.

En los casos más severos de hiperparatiroidismo primario se puede presentar calcificación del tejido renal (nefrocalcinosis), la cual puede ser detectada mediante técnicas convencionales de rayos $X$; sin embargo, los pequeños depósitos de fosfato cálcico en las papilas renales (placas de Randall) no son visibles radiológicamente, pero parecen estar implicadas en la nefrocalcinosis [23].

\section{Manifestaciones en el sistema cardiovascular}

En la época en la que la presentación sintomática del hiperparatiroidismo primario era la principal forma clínica existía una clara relación con un incremento en la mortalidad cardiovascular, mientras su relación con la forma asintomática es más incierta. El hallazgo electrocardiográfico más frecuentemente reportado con la hipercalcemia es el acortamiento del intervalo QT lo cual pudiera favorecer la aparición de arritmias [24].

Existen algunas evidencias observacionales y estudios pobremente controlados que sugieren que el hiperparatiroidismo primario, especialmente su forma sintomática, y los niveles de la paratohormona se pueden relacionar con calcificación coronaria y de la válvula aórtica; no obstante, la corrección de la enfermedad pareciera no influir en estos desenlaces. Hasta no tener mayor y mejor evidencia no se puede asegurar que el hiperparatiroidismo primario tenga algún impacto en el corazón [21].

Algo similar ocurre con la enfermedad de los grandes vasos; se ha propuesto que el hiperparatiroidismo primario se pudiera relacionar con mayor rigidez vascular [25] o empeorar las placas ateroescleróticas carotideas [26]; sin embargo, no es posible asegurar que estos hallazgos se asocien a un mayor riesgo cardiovascular.

\section{Manifestaciones neuropsicológicas y cognitivas}

El hiperparatiroidismo primario sintomático ha sido relacionado por años con síntomas neuropsicológicos como depresión, ansiedad, cambios en la memoria e iniciativa, irritabilidad y alteraciones en el sueño; todo esto tiene plausibilidad debido al papel del calcio en los diferentes procesos neurológicos. Pese a esto, los pocos estudios publicados con un diseño sólido han reportado datos inconsistentes respecto a los cambios en la sintomatología después de la cirugía [27].

Estudios observacionales relacionan tanto el hiperparatiroidismo primario sintomático como el asintomático con mayor riesgo de depresión y otras formas de enfermedad mental, los cuales parecieran estar relacionados con los niveles de calcio, e indican que al menos los síntomas de depresión, mas no los de ansiedad, pudieran mejorar después de la cirugía [28-30]. El hiperparatiroidismo primario se asocia en diferentes estudios con alteraciones en diferentes áreas cognitivas como la memoria visual y verbal, la memoria y la coordinación, así como el procesamiento de la información; sin embargo, se reportan distintos sitios comprometidos y no en todos existe mejoría con la cirugía [21]. 


\section{Hiperparatiroidismo primario normocalcémico}

El hiperparatiroidismo primario normocalcémico se define por las concentraciones consistentemente elevadas de la paratohormona, con una concentración de calcio sérico total e ionizado normales y en ausencia de otras posibles causas que puedan elevar la paratohormona. Esta forma no fue reconocida hasta el 2008 [27] y su fisiopatología sigue siendo pobremente entendida; se sospecha que pueda existir una resistencia tisular al efecto de la paratohormona [31]. Para establecer el diagnóstico de un hiperparatiroidismo primario normocalcémico se deben tener en cuenta ciertas condiciones [32,33]:

Existe la posibilidad de que los pacientes con hiperparatiroidismo primario con hipercalcemia en ocasiones tengan niveles de calcio normales, por esto no se debe confiar en una sola medición de calcio.

- Los niveles de 25-hidroxi vitamina D deben ser superiores a $20 \mathrm{ng} / \mathrm{mL}$, idealmente superiores a $30 \mathrm{ng} / \mathrm{mL}$. Si bien no es claro que valores inferiores se asocian con el incremento en el nivel de la paratohormona algunos pacientes con calcio normal pueden desarrollar hipercalcemia al normalizar los valores de vitamina D.

- La filtración glomerular debe ser superior a $60 \mathrm{~mL} / \mathrm{min}$ ya que los niveles de la paratohormona comienzan a subir con una disminución en la depuración por debajo de este valor.

No se puede establecer con seguridad en aquellos pacientes que se encuentren en tratamiento con diuréticos tiazídicos, litio, denosumab o bifosfonatos, ya que todos estos medicamentos se han asociado con incrementos variables de la paratohormona.

No es posible confirmarlo cuando se documenta hipercalciuria, ya que un defecto renal primario que produzca hipercalciuria puede incrementar los valores de la paratohormona.

Considerar si existen trastornos gastrointestinales asociados a malabsorción de calcio, los cuales se pueden presentar algunas veces sin obvias manifestaciones gastrointestinales.

La mayoría de los reportes del hiperparatiroidismo primario normocalcémico provienen de centros en los cuales los pacientes fueron evaluados por enfermedad metabólica ósea. Esto pudiera explicar la alta frecuencia con la que se reporta osteoporosis (en promedio $48 \%$ ), fracturas por fragilidad (en promedio $17 \%$ ) y nefrolitiasis (en promedio 17\%) [34]. Cuando se han comparado los pacientes con hiperparatiroidismo primario asintomático y aquellos con hiperparatiroidismo primario normocalcémico pareciera no existir diferencia en el riesgo de presentar fracturas por fragilidad y nefrolitiasis [35].

Respecto a la prevalencia del hiperparatiroidismo primario normocalcémico los datos son bastante discordantes entre los diversos estudios, donde se encuentran valores entre $0,4 \%$ y $17 \%$, similar a lo reportado en el hiperparatiroidismo primario [34]. Además, se ha documentado que estos pacientes pueden progresar a una forma sintomática - hipercalcémica de hiperparatiroidismo primario, pero nuevamente los datos publicados son bastante heterogéneos con frecuencias de progresión entre $0 \%$ y $40 \%$ [1]. 
El hiperparatiroidismo primario normocalcémico es una condición pobremente entendida que algunos autores sugieren no debería ser considerada como una forma leve de hiperparatiroidismo primario ni una forma asintomática de hiperparatiroidismo primario, ya que algunos pacientes presentan manifestaciones en órganos como el hueso y el riñón. El seguimiento de estos pacientes es incierto ya que no es posible predecir quienes progresaran a una forma hipercalcémica ni quienes desarrollarán una forma sintomática. El seguimiento propuesto es el mismo que para el hiperparatiroidismo primario asintomático (véase tabla 1) [15].

\section{Hiperparatiroidismo primario agudo}

El criterio para diagnosticar un hiperparatiroidismo primario agudo por intoxicación paratiroidea, envenenamiento paratiroideo, tormenta paratiroidea o crisis hiperparatiroidea se basa usualmente en una concentración de calcio sérico por encima de 14$15 \mathrm{mg} / \mathrm{dL}$, asociado a los síntomas y signos de hipercalcemia descritos previamente. La posibilidad de desarrollar una crisis hipercalcemia pareciera ser rara en el hiperparatiroidismo primario, donde se encuentran valores inferiores al $1 \%$ [36].

No se ha descubierto ningún factor que permita predecir el desarrollo de una crisis hipercalcémica, y su distribución pareciera seguir las mismas características del hiperparatiroidismo primario. Los reportes de pacientes con hiperparatiroidismo primario agudo se caracterizan por niveles aumentados de calcio, con valores tan altos como $26,3 \mathrm{mg} / \mathrm{dL}$, y de paratohormona de hasta 20 veces superiores a los rangos de referencia. Las concentraciones incrementadas de calcio y paratohormona deben hacer sos- pechar de un hiperparatiroidismo primario agudo o un carcinoma de paratiroides, este último usualmente con valores alrededor de 10 veces los de los rangos de referencia, por lo que algunos autores sugieren que el valor de la paratohormona puede ser útil para diferenciar ambas condiciones [37].

Generalmente los pacientes con hiperparatiroidismo primario agudo cursan con compromiso renal y esquelético en proporciones mayores a aquellas reportadas en personas con hiperparatiroidismo primario, usualmente con calciurias de hasta 2 g/día [37]; además, se ha reportado mayor frecuencia de pancreatitis en los primeros respecto a los segundos. En un reporte de un paciente con hiperparatiroidismo primario agudo, a quien le realizaron una biopsia ósea, se describen cambios celulares muy interesantes que hacen pensar que en esta condición hay un aumento en el fenómeno de resorción mas no en el de formación, lo que lo diferenciaría del hiperparatiroidismo primario, en el cual ambos procesos óseos se encuentran aumentados [38].

\section{Diagnóstico}

Como se mencionó previamente el cuadro bioquímico clásico del hiperparatiroidismo primario se caracteriza por concentraciones elevadas de la paratohormona con altos niveles de calcio y bajos de fósforo en suero, aumento en la concentración de la 1,25-dixidroxi vitamina $D$ e hipercalciuria. Dado que la forma más prevalente es la asintomática, su descubrimiento frecuentemente es fortuito, por ejemplo, durante una medición rutinaria de calcio por alguna sintomatología inespecífica o dentro del estudio de algún trastorno metabólico óseo; rara vez dentro del estudio de la urolitiasis. 
En reportes de varios países las crisis hipercalcémicas asociadas con un hiperparatiroidismo primario son consideradas exóticas [1], pero en nuestro medio, a pesar de que no son muy frecuentes, sí se pueden presentar más a menudo, aunque no hay estudios que apoyen o refuten esto.

\section{Medición de la paratohormona}

Actualmente, la medición de la paratohormona se lleva a cabo mediante inmunoensayo. La primera generación de estos ensayos presentaba bastantes dificultades para diferenciar entre individuos sanos, personas con hipercalcemia de origen diferente a un hiperparatiroidismo primario y un verdadero hiperparatiroidismo primario. Afortunadamente los ensayos de segunda (los más ampliamente disponibles) y de tercera generación tienen un rendimiento diagnóstico mucho mejor [27].

Los inmunoensayos de segunda generación tienen una mayor especificidad para detectar la paratohormona intacta en comparación con los de primera (1-84); sin embargo, puede presentar reacción cruzada con los fragmentos amino truncados ( $\mathrm{N}$-truncados o paratohormona no 1-84) de los cuales el más frecuente es la paratohormona 7-84. Los ensayos de tercera generación usan anticuerpos contra la paratohormona 1-4, lo que evita la reacción cruzada con los fragmentos $\mathrm{N}$-truncados; además, los ensayos de tercera generación son capaces de detectar la N-paratohormona, una forma de paratohormona con una modificación postraduccional que representa el $10 \%$ de la paratohormona en individuos sanos y hasta el $15 \%$ en personas con enfermedad renal y cuya producción se encuentra muy aumentada en condiciones como el hiperparatiroidismo primario severo y el carcinoma paratiroideo [39].

\section{Medición de calcio}

Las concentraciones séricas de calcio en un adulto generalmente varían entre $8,6 \mathrm{mg} /$ $\mathrm{dL}$ y $10,4 \mathrm{mg} / \mathrm{dL}$. Alrededor del $45 \%$ se encuentra unido a las proteínas plasmáticas y otro $10 \%$ a aniones como fosfato y citrato; el calcio ionizado o libre $(1,17 \mathrm{mmol} / \mathrm{L}$ a $1,33 \mathrm{mmol} / \mathrm{L})$ representa el $45 \%$ restante del calcio circulante. El calcio ionizado es el biológicamente disponible y tiende a permanecer estable debido a la fina regulación que hace la paratohormona; por su parte, el calcio unido a proteínas varía en función de la concentración de las mismas [32]. No obstante, la recomendación sigue siendo utilizar el valor de calcio corregido derivado de la fórmula:

\section{calcio corregido = calcio sérico total $(\mathrm{mg} / \mathrm{L})$ $+0,8 \times$ (4-albúmina ( $g / d L)$}

Esta directriz se basa en que la mayoría de los centros médicos no tienen la capacidad de hacer una medición confiable del calcio ionizado [15].

La medición de calcio se debe realizar preferiblemente a partir de una muestra tomada de una vena por la cual la sangre fluya libremente, ya que la estasis venosa, debido al uso del torniquete, puede ocasionar pérdida de líquido a través de la pared vascular, lo que incrementa los niveles de calcio unido a proteínas. El análisis no se debe demorar y se debe practicar en los primeros 30 minutos de extraída la sangre ya que los cambios en el $\mathrm{pH}$ alteran las concentraciones de calcio libre. Finalmente, la muestra se debe tomar de sangre total sin anticoagulantes como citrato, oxalato o EDTA para evitar precipitaciones que pueden resultar en valores falsamente bajos de calcio [40]. 


\section{Medición de 25-hidroxi vitamina $\mathrm{D}$}

La deficiencia de 25-hidroxi vitamina $D$ es frecuente en los pacientes con hiperparatiroidismo primario y, probablemente, sea debido al incremento en la depuración mediada por la 1,25-dihidroxi vitamina $D$ y la paratohormona; no obstante, la deficiencia de 25-hidroxi vitamina $D$ es un hallazgo bastante común en la población general [41]. Los niveles bajos de 25-hidroxi vitamina $D$ se han asociado con un mayor tamaño en los adenomas y una alta probabilidad que en la gammagrafía de paratiroides se reporte una alteración [32].

Después de la resolución del hiperparatiroidismo primario los niveles de 25-hidroxi vitamina $D$ regresan a los valores normales, y con la suplementación puede tomar entre seis y doce meses para que los niveles de la paratohormona vuelvan a la normalidad. El reemplazo con vitamina $D$ en personas con hiperparatiroidismo primario no pareciera incrementar el riesgo de hipercalcemia [42]. La medición de 1,25-dihidroxi vitamina $D$ no es necesaria de manera rutinaria en el estudio del paciente con hiperparatiroidismo primario.

\section{Estudio genético}

El hiperparatiroidismo primario puede presentarse como una condición aislada o esporádica, como parte de un síndrome familiar complejo como la neoplasia endocrina múltiple (tipo 1 a 4) o como una forma familiar aislada. De esta manera, se ha propuesto un algoritmo para el enfoque diagnóstico genético en pacientes con hiperparatiroidismo primario (véase figura 3) $[32,43]$.

\section{Localización preoperatoria}

El $90 \%$ de los casos de hiperparatiroidismo primario se explica por un adenoma único, alrededor del $10 \%$ tiene enfermedad multiglandular y es infrecuente encontrar un carcinoma de paratiroides. Tradicionalmente, el tratamiento del hiperparatiroidismo primario se hacía mediante la exploración quirúrgica cervical bilateral que permitía la visualización de las cuatro glándulas. Desde finales del siglo xx la paratiroidectomía selectiva o mínimamente invasiva se convirtió en la modalidad más utilizada debido a un mayor uso y a los avances alcanzados en la tecnología de localización [44].

La localización preoperatoria es importante debido a que hasta en el $15 \%$ de los casos las glándulas hiperfuncionantes pueden tener una localización inusual, las glándulas superiores (que derivan del cuarto arco branquial) pueden localizarse dentro de la glándula tiroides, las glándulas inferiores (que provienen del tercer arco branquial) descienden con el timo $y$, al tener una migración mayor, pueden tener una localización final mucho más variada desde el ángulo de la mandíbula anterior al nervio laríngeo recurrente hasta el pericardio, por lo cual se pueden encontrar en el tracto tirotímico o en el mediastino [45]. La gammagrafía con ${ }^{99 m}$ Tc sestamibi y la ecografía de cuello son probablemente las ayudas diagnósticas más utilizadas. La tomografía dinámica, la resonancia magnética y la tomografía por emisión de positrones (PET) son opciones generalmente reservadas para situaciones especiales [46].

\section{Ecografía}

Esta técnica ofrece muchas ventajas: su costo es bajo, está ampliamente disponible y su sensibilidad (76\%) y valor predictivo po- 


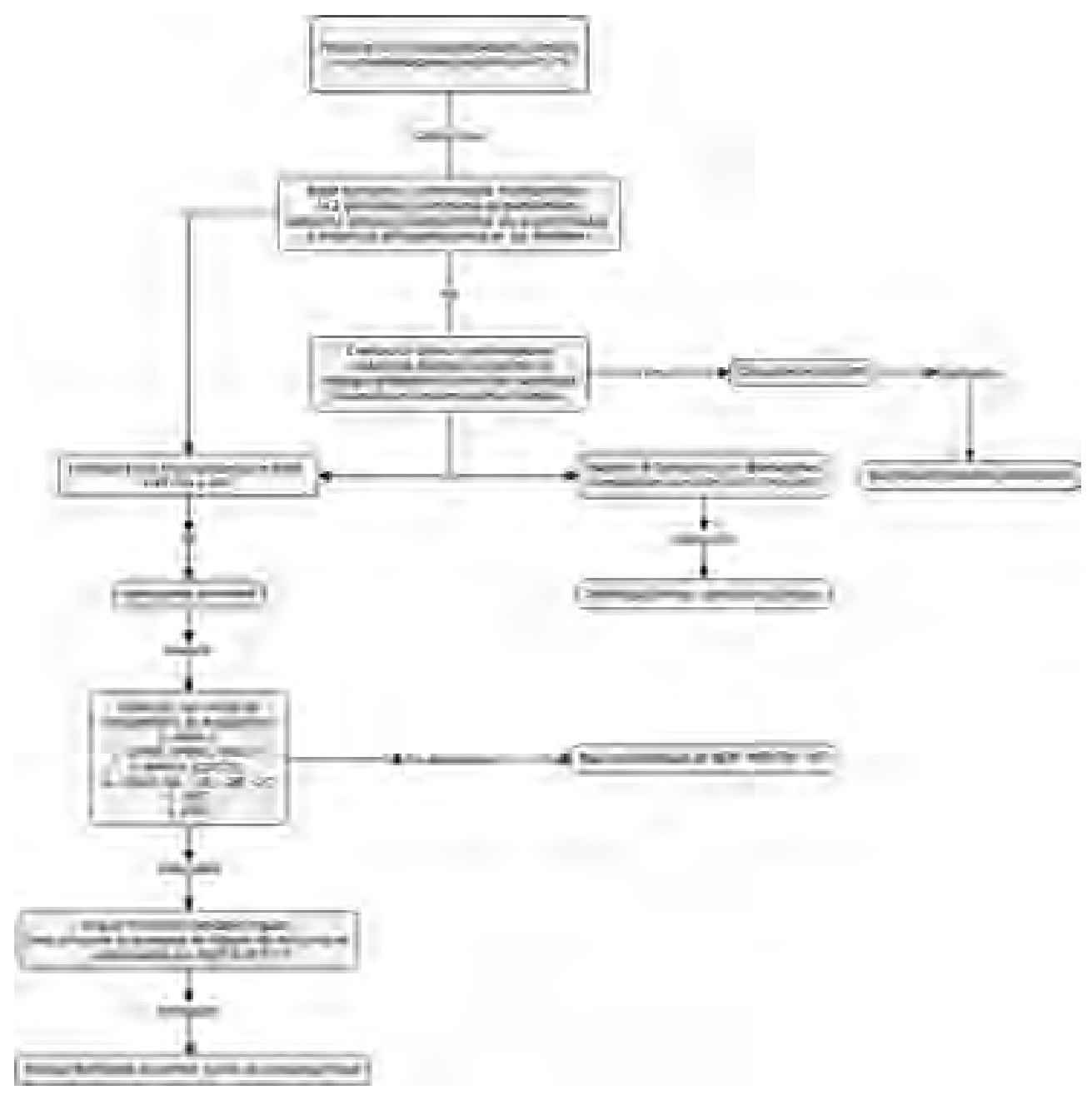

Figura 3. Aproximación al estudio genético del paciente con hiperparatiroidismo primario. NEM = neoplasia endocrina múltiple. HPT-TM = síndrome de hiperparatiroidismo-tumor de mandíbula, HFF = hipercalcemia hipocalciúrica familiar, HPE= hiperparatiroidismo primario esporádico, HPAF = hiperparatiroidismo primario aislado familiar. *Se considera joven a aquella persona menor de 30 años; sin embargo, el 10\% de las menores de 45 años con hiperparatiroidismo primario tienen una mutación. Tomado y adaptado de "Diagnosis of Asymptomatic Primary Hyperparathyroidism: Proceedings of the Fourth International Workshop" por Eastell y colaboradores, 2014, J Clin Endocrinol Metab, 99, p. 3577. Copyright (C) 2014 por Endocrine Society [32] y de "Familial and Hereditary Forms of Primary Hyperparathyroidism" por R.V. Thakker. En: The Parathyroids (p. 350) por Bilezikian JP y colaboradores, eds., 2015, California, Estados Unidos: Academic Press. Copyright (C2015 Elsevier Inc [43].

sitivo $(93,2 \%)$ son bastante aceptables para una prueba de detección inicial [47]. La enfermedad multiglandular y las lesiones tiroideas pueden impactar significativamente en su desempeño. En un reporte con más de
20.000 casos se mostró cómo la sensibilidad caía de un $78,5 \%$ a un $35 \%$ en los casos de enfermedad multiglandular, y a un $16 \%$ en los de doble adenoma [48]. Como en todas las ecografías la experiencia del operador es 
fundamental y se deben tener en cuenta varios aspectos técnicos como la cantidad de grasa subcutánea en el cuello o la localización ectópica de las glándulas, especialmente las localizadas en el mediastino; es por esto que rara vez se utiliza sola.

\section{Gammagrafía con ${ }^{99 m}$ Tc sestamibi}

Esta técnica se ha utilizado desde la primera mitad del siglo $\mathrm{xx}$, inicialmente con Talio y posteriormente con ${ }^{99 \mathrm{~m}} \mathrm{Tc}$ sestamibi, el cual aumentó considerablemente la sensibilidad. $\mathrm{El}{ }^{99 \mathrm{~m}} \mathrm{TC}$ se acumula en las mitocondrias que abundan en las células de los adenomas de paratiroides. La mayoría de los protocolos se basan en dos técnicas, la gammagrafía de doble fase con un solo trazador o la gammagrafía de fase única con doble trazador [49].

En la técnica con un solo trazador con doble fase el ${ }^{99 m} \mathrm{Tc}$ sestamibi se administra y se toman las imágenes iniciales 10 a 15 minutos después, y las posteriores entre 1,5 y 3 horas después. El radiotrazador desaparece más rápido de los tejidos circundantes que de las paratiroides, lo que permite la identificación de las glándulas anormales. En la técnica de fase única con doble trazador, conocida también como gammagrafía de substracción, se utiliza un segundo trazador que es captado con mayor avidez por la glándula tiroides, posteriormente, las imágenes se pueden substraer digitalmente o pueden ser evaluadas de manera simultánea para detectar las lesiones en las glándulas paratiroides [50].

En comparación con la ecografía esta técnica presenta varias ventajas; su amplio campo de evaluación permite detectar lesiones localizadas en zonas diferentes al cuello y presenta menor variabilidad interobservador. Sin embargo, tiene el potencial de arrojar falsos positivos y falsos negativos, los primeros en los casos con nódulos tiroi- deos que tengan una depuración del trazador más lenta y los segundos en los de enfermedad multiglandular y en personas con enfermedad renal crónica. La sensibilidad reportada varía entre los estudios, pero se encuentra alrededor del 70\% [51].

En relación con las imágenes planares, la tomografía de emisión por fotón único (SPECT; del inglés, Single Photon Emission Computed Tomography) tiene la ventaja de la tridimensionalidad, la cual permite una mejor evaluación del espacio retrotiroideo que aumenta la sensibilidad de la prueba [52]. Recientemente se ha comenzado a utilizar la fusión entre la tomografía de emisión por fotón único y la tomografía convencional (SPECT/CT), lo cual no solo permite tener una mejor información anatómica, sino que aumenta considerablemente la sensibilidad y el valor predictivo positivo ( $84 \%$ y $95 \%$, respectivamente) [53].

Entonces ¿cuál usar como imagen inicial, la gammagrafía o la ecografía? Si bien ambas técnicas tienen sus ventajas y en algunos centros se acostumbra usarlas simultáneamente, cuando se comparan la ecografía y la gammagrafía planar parecen tener un rendimiento diagnóstico similar; sin embargo, la tomografía de emisión por fotón único, sola o fusionada con la tomografía convencional, puede ser algo superior, incluso para detectar las glándulas localizadas en el timo, el espacio retroesternal o retroesofágico. Por lo tanto, si se cuenta con alguna de estas técnicas deberían preferirse como la imagen inicial. En la tabla 2 se resumen algunos estudios que comparan ambas técnicas.

\section{Tomografía computarizada}

Comparada con otras técnicas la sensibilidad de la tomografía computarizada (CT) es inferior. La tomografía dinámica contrastada 


$\begin{aligned} & \text { Tabla 2. Ecografía frente a gammagrafía para la localización perioperatoria de un adenoma } \\
& \text { paratiroideo }\end{aligned}$
\begin{tabular}{lllll}
\multicolumn{1}{c}{ Referencia } & Ecografía & \multicolumn{3}{c}{ Técnica } \\
& Sensibilidad & VPP & Sensibilidad & VPP \\
\hline Kluijfhout y colaboradores (2015) [55] & $63 \%$ & $78 \%$ & $80 \%$ & $93 \%$ \\
Guerin y colaboradores (2015) [56] & $84,5 \%$ & $78,5 \%$ & $93 \%$ & $86 \%$ \\
Berner y colaboradores (2015) [57] & $60,3 \%$ & $89 \%$ & $70,6 \%$ & $98 \%$ \\
VPP=valor predictivo positivo & & & & \\
\hline
\end{tabular}

permite resolver algunas de las dificultades de la tomografía computarizada convencional por medio de la incorporación de dos fases contrastadas a la fase no contrastada. Usualmente los adenomas paratiroideos tienen baja atenuación en la fase no contrastada y alcanzan el pico máximo de realce en la fase arterial con un posterior lavado del contraste en las fases más tardías; esto permite diferenciarlos de los ganglios linfáticos que muestran un realce más persistente en las fases tardías. En relación con la gammagrafía plana y la ecografía la tomografía computarizada dinámica pareciera tener una mejor sensibilidad [57]. Sin embargo, esta técnica expone al paciente a una dosis considerable de radiación. Mahajan y colaboradores (2012) [58] reportaron que, a pesar de tener la misma dosis efectiva de radiación que una SPECT, con la tomografía computarizada dinámica la dosis a la tiroides era 57 veces mayor.

\section{Resonancia magnética}

Aunque se usa con menos frecuencia, la resonancia magnética tiene una sensibilidad similar a la de otras técnicas [59]. Al ser los adenomas paratiroideos lesiones altamente vascularizadas usualmente se observan hiperintensos en las imágenes ponderadas en $\mathrm{T} 2$ e hipointensas en las ponderadas en T1. Aprovechando las características de esta técnica se ha explorado la utilidad de la resonancia magnética dinámica, la cual ha mostrado resultados promisorios [60].

\section{Tomografía por emisión de positrones-tomografía computada}

El uso de la tomografía por emisión de positrones-tomografía computada (PET/CT) se ha limitado al ámbito académico debido a su alto costo y baja disponibilidad. Sin embargo, la mejor resolución espacial y el bajo tiempo de adquisición de las imágenes, así como el desarrollo de nuevos trazadores, la hacen una técnica interesante. De todas las opciones disponibles aquella con ${ }^{18 F}$-fluorocolina pareciera ser la más prometedora [61].

\section{Tratamiento}

La resección quirúrgica del tejido paratiroideo hiperfuncionante es la única forma de cura definitiva para el hiperparatiroidismo primario y la paratiroidectomía mínimamente invasiva; es la técnica preferida por la mayoría de los cirujanos ya que se asocia a un menor tiempo quirúrgico, menores complicaciones posquirúrgicas y a una mayor satisfacción del paciente [62].

Todos los pacientes con un hiperparatiroidismo primario sintomático deberían ser 
tratados quirúrgicamente, y aquellos en que se logre la identificación preoperatoria de un adenoma paratiroideo, de acuerdo a la experiencia y disponibilidad del centro asistencial, deberían ser tratados con una paratiroidectomía mínimamente invasiva. Existen recomendaciones para elegir el tratamiento quirúrgico en los pacientes asintomáticos (véase tabla 1) y estas se extienden a los pacientes con un hiperparatiroidismo primario normocalcémico. Está por fuera de los objetivos de este capítulo hacer una descripción detallada de las técnicas quirúrgicas disponibles.

En los pacientes con hiperparatiroidismo primario asintomático, en quienes a pesar de cumplir con las condiciones propuestas para el manejo quirúrgico se decide no realizar la intervención quirúrgica, bien sea por comorbilidades, contraindicaciones o deseo del paciente, existen algunas alternativas para el manejo médico.

\section{Suplementación de calcio y vitamina D}

El manejo de la dieta del paciente con hiperparatiroidismo primario es controvertido debido a la poca evidencia científica disponible. El consumo de calcio en la dieta afecta los niveles de la paratohormona; una baja ingesta los incrementa, incluso se ha mostrado en modelos animales que una ingesta crónica baja de calcio favorece la aparición de hiperplasia de las glándulas paratiroides [63]. La suplementación de calcio en personas con hiperparatiroidismo primario y baja ingesta pareciera disminuir los niveles de la paratohormona, pero la evidencia es insuficiente para recomendar ampliamente su implementación [64]. No hay ninguna razón para recomendar una ingesta diferente de calcio en las personas con hiperparatiroidismo primario.
Los niveles de vitamina $D$ en personas con hiperparatiroidismo primario varían alrededor del mundo dependiendo, probablemente, de factores como la exposición solar, el estado nutricional o la posibilidad de acceder a los servicios de salud [32]. En personas con hiperparatiroidismo primario asintomático los niveles bajos de vitamina $D$ se han asociado con un mayor recambio óseo y, en algunos estudios, con mayor riesgo cardiovascular [65]. Los niveles bajos de vitamina $D$ son consecuencia, en menor medida, de la conversión a la 1,25-dihidroxi vitamina D y, en mayor medida, a la inactivación por la enzima 24-hidroxilasa. El reemplazo de la vitamina $D$ pareciera disminuir los niveles de la paratohormona [66], pero pudiera aumentar los niveles de calcio en suero o la excreción de calcio urinario en algunos pacientes. Por esto, en caso de decidir suplementarla, el paciente debe ser vigilado estrechamente. La deficiencia de vitamina D aumenta el riesgo de hipocalcemia posoperatoria y, por tal razón, debería reponerse antes de la cirugía [67].

\section{Estrógeno}

Algunos estudios observacionales han sugerido un posible efecto en la disminución del calcio con la aplicación de estrógeno; sin embargo, los ensayos clínicos no lo confirmaron. Su administración mejora la densidad mineral ósea sin disminuir el riesgo de fracturas no vertebrales; efecto que también se ha demostrado en pacientes posmenopáusicas sin hiperparatiroidismo primario [15].

\section{Bifosfonatos}

El alendronato ha sido el bifosfonato más utilizado para el hiperparatiroidismo primario. Tanto en hombres como mujeres ha demostrado disminuir los niveles de calcio, 
aumentar la densidad mineral ósea en columna y cadera y disminuir los marcadores de resorción $[68,69]$. La evidencia con otros bifosfonatos es limitada.

\section{Calcimiméticos}

El cinacalcet es un activador alostérico del receptor sensible al calcio que ha sido utilizado en pacientes con hiperparatiroidismo primario terciario, hipercalcemia por carcinoma de paratiroides e hiperparatiroidismo primario. En el hiperparatiroidismo primario su uso se reserva para aquellos pacientes con indicación de cirugía y que no se les puede realizar o en caso de hipercalcemia posoperatoria persistente. Varios ensayos clínicos han evaluado la utilidad del cinacalcet en el hiperparatiroidismo primario (véase tabla $\mathbf{3}$ ), los cuales han demostrado disminución en los niveles de calcio y la paratohormona; no obstante, no existen datos consistentes en la reducción del riesgo de daño orgánico (p. ej. osteoporosis, fracturas, urolitiasis, disfunción renal). En otros países el costo del cinacalcet lo hace una opción que no es costo-efectiva cuando se compara con la paratiroidectomía [70], por lo cual se reserva solo para casos seleccionados de hipercalcemia intratable con cirugía [65].

\begin{tabular}{|c|c|c|}
\hline \multirow{2}{*}{ Referencia } & \multicolumn{2}{|c|}{ Desenlaces } \\
\hline & $\begin{array}{l}\text { Disminución } \\
\text { del calcio }\end{array}$ & $\begin{array}{l}\text { Disminución } \\
\text { de la parato- } \\
\text { hormona }\end{array}$ \\
\hline $\begin{array}{l}\text { Shoback y colabo- } \\
\text { radores (2003) [72] }\end{array}$ & $18,5 \%$ & $50 \%$ \\
\hline $\begin{array}{l}\text { Peacock y colabo- } \\
\text { radores (2009) [73] }\end{array}$ & $12 \%$ & $19 \%$ \\
\hline $\begin{array}{l}\text { Khan y colabora- } \\
\text { dores (2015) [74] }\end{array}$ & $15 \%$ & $23,8 \%$ \\
\hline
\end{tabular}

\section{Conclusiones}

El hiperparatiroidismo primario en su forma sintomática pareciera ser una condición poco frecuente hoy en día; sin embargo, la disponibilidad de métodos más confiables, rápidos y menos costosos para medir el calcio han hecho de la forma asintomática una endocrinopatía bastante común. Aún no es clara la forma en que un hiperparatiroidismo primario asintomático evoluciona a uno sintomático, pero este último se asocia a alteraciones en la salud ósea y renal, y posiblemente esté implicado en alteraciones en otros sistemas como el cardiovascular y el sistema nervioso central. Sin tratamiento esta alteración puede llegar a poner en riesgo la vida del paciente o dejar secuelas permanentes.

De una correcta interpretación de las mediciones de calcio, fósforo, albúmina, paratohormona y vitamina $D$ dependerá el diagnóstico oportuno y acertado, al igual que una selección cuidadosa de la prueba de localización puede ayudar a hacer el tratamiento quirúrgico menos extenso y más exitoso. Sin embargo, no todos los pacientes se beneficiarán de este. Por lo tanto, se deben seleccionar muy bien aquellas personas que serán llevadas al quirófano.

Afortunadamente, la mayoría de casos de hiperparatiroidismo primario pueden curarse sin secuelas permanentes y, en manos de un cirujano experto, el riesgo de complicaciones es mínimo.

\section{Agradecimientos}

A los doctores Carlos Simón Duque Fisher, MD, y Carolina Prieto Saldarriaga, MD, por las valiosas sugerencias y revisión del manuscrito terminado. 


\section{Bibliografía}

1. Bandeira L, Bilezikian J. Primary Hyperparathyroidism. F1000Res 2016; 5.

2. Albright F, Aub JC, Bauer W. Hyperparathyroidism: A common and polymorphic condition as illustrated by seventeen proved cases from one clinic. JAMA 1934; 102: 1276-1287.

3. Cusano NE, Silverberg SJ, Bilezikian JP. Normocalcemic primary hyperparathyroidism. J Clin Densitom 2013; 16: 33-39.

4. Keating FR, Jr. Diagnosis of primary hyperparathyroidism. Clinical and laboratory aspects. JAMA 1961; 178: 547555.

5. Eufrazino C, Veras A, Bandeira F. Epidemiology of Primary Hyperparathyroidism and its Non-classical Manifestations in the City of Recife, Brazil. Clin Med Insights Endocrinol Diabetes 2013; 6: 69-74.

6. Gasser RW. Clinical aspects of primary hyperparathyroidism: clinical manifestations, diagnosis, and therapy. Wien Med Wochenschr 2013; 163: 397-402.

7. Wermers RA, Khosla S, Atkinson EJ, Achenbach SJ, Oberg AL, Grant CS, et al. Incidence of primary hyperparathyroidism in Rochester, Minnesota, 1993-2001: an update on the changing epidemiology of the disease. J Bone Miner Res 2006; 21: 171-177.

8. Lassen T, Friis-Hansen L, Rasmussen AK, Knigge U, Feldt-Rasmussen $\mathbf{U}$. Primary hyperparathyroidism in young people. When should we perform genetic testing for multiple endocrine neoplasia 1 (MEN-1)? J Clin Endocrinol Metab 2014; 99: 3983-3987.

9. Roizen J, Levine MA. A meta-analysis comparing the biochemistry of primary hyperparathyroidism in youths to the biochemistry of primary hyperparathyroidism in adults. J Clin Endocrinol Metab 2014; 99: 4555-4564.

10. Cohen J, Gierlowski TC, Schneider AB. A prospective study of hyperparathyroidism in individuals exposed to radiation in childhood. Jama 1990; 264: 581-584.

11. Wermers RA, Kearns AE, Jenkins GD, Melton LJ, 3rd. Incidence and clinical spectrum of thiazide-associated hypercalcemia. Am J Med 2007; 120: 911.e919-915.

12. McHenry CR, Lee K. Lithium therapy and disorders of the parathyroid glands. Endocr Pract 1996; 2: 103-109.

13. Liu JM, Cusano NE, Silva BC, Zhao L, He XY, Tao B, et al. Primary Hyperparathyroidism: A Tale of Two Cities Revisited - New York and Shanghai. Bone Res 2013; 1: 162-169.

14. Yeh MW, Ituarte PH, Zhou HC, Nishimoto S, Liu IL, Harari $\mathbf{A}$, et al. Incidence and prevalence of primary hyperparathyroidism in a racially mixed population. J Clin Endocrinol Metab 2013; 98: 1122-1129.

15. Bilezikian JP, Brandi ML, Eastell R, Silverberg SJ, Udelsman R, Marcocci C, et al. Guidelines for the management of asymptomatic primary hyperparathyroidism: summary statement from the Fourth International Workshop. J Clin Endocrinol Metab 2014; 99: 3561-3569.

16. Rejnmark L, Amstrup AK, Mollerup CL, Heickendorff L, Mosekilde L. Further insights into the pathogenesis of primary hyperparathyroidism: a nested case-control study. J Clin Endocrinol Metab 2013; 98: 87-96.

17. Vignali E, Viccica G, Diacinti D, Cetani F, Cianferotti L, Ambrogini E, et al. Morphometric vertebral fractures in postmenopausal women with primary hyperparathyroidism. J Clin Endocrinol Metab 2009; 94: 2306-2312.

18. Cipriani C, Biamonte F, Costa AG, Zhang C, Biondi P, Diacinti D, et al. Prevalence of kidney stones and vertebral fractures in primary hyperparathyroidism using imaging technology. J Clin Endocrinol Metab 2015; 100: 1309-1315.

19. Kulak CA, Bandeira C, Voss D, Sobieszczyk SM, Silverberg SJ, Bandeira F, et al. Marked improvement in bone mass after parathyroidectomy in osteitis fibrosa cystica. J Clin Endocrinol Metab 1998; 83: 732-735.

20. Bandeira F, Griz L, Caldas G, Bandeira C, Freese E. From mild to severe primary hyperparathyroidism: The Brazilian experience. Arq Bras Endocrinol Metabol 2006; 50: 657663.

21. Silverberg SJ, Clarke BL, Peacock M, Bandeira F, Boutroy S, Cusano NE, et al. Current issues in the presentation of asymptomatic primary hyperparathyroidism: proceedings of the Fourth International Workshop. J Clin Endocrinol Metab 2014; 99: 3580-3594.

22. Sorensen MD, Duh QY, Grogan RH, Tran TC, Stoller ML. Urinary parameters as predictors of primary hyperparathyroidism in patients with nephrolithiasis. J Urol 2012; 187: 516-521.

23. Evan AE, Lingeman JE, Coe FL, Miller NL, Bledsoe SB, Sommer AJ, et al. Histopathology and surgical anatomy of patients with primary hyperparathyroidism and calcium phosphate stones. Kidney Int 2008; 74: 223-229.

24. Bilezikian JP. Management of acute hypercalcemia. N Engl J Med 1992; 326: 1196-1203.

25. Rubin MR, Maurer MS, McMahon DJ, Bilezikian JP, Silverberg SJ. Arterial stiffness in mild primary hyperparathyroidism. J Clin Endocrinol Metab 2005; 90: 3326-3330.

26. Walker MD, Fleischer J, Rundek T, McMahon DJ, Homma S, Sacco R, et al. Carotid vascular abnormalities in primary hyperparathyroidism. J Clin Endocrinol Metab 2009; 94: 3849-3856.

27. Bilezikian JP, Khan AA, Potts JT, Jr. Guidelines for the management of asymptomatic primary hyperparathyroidism: summary statement from the third international workshop. J Clin Endocrinol Metab 2009; 94: 335-339.

28. Yu N, Leese GP, Donnan PT. What predicts adverse outcomes in untreated primary hyperparathyroidism? The Parathyroid Epidemiology and Audit Research Study (PEARS). Clin Endocrinol (Oxf) 2013; 79: 27-34.

29. Espiritu RP, Kearns AE, Vickers KS, Grant C, Ryu E, Wermers RA. Depression in primary hyperparathyroidism: 
prevalence and benefit of surgery. J Clin Endocrinol Metab 2011; 96: E1737-1745.

30. Kahal H, Aye M, Rigby AS, Sathyapalan T, England RJ, Atkin SL. The effect of parathyroidectomy on neuropsychological symptoms and biochemical parameters in patients with asymptomatic primary hyperparathyroidism. Clin Endocrinol (Oxf) 2012; 76: 196-200.

31. Maruani G, Hertig A, Paillard M, Houillier P. Normocalcemic primary hyperparathyroidism: evidence for a generalized target-tissue resistance to parathyroid hormone. J Clin Endocrinol Metab 2003; 88: 4641-4648.

32. Eastell R, Brandi ML, Costa AG, D'Amour P, Shoback DM, Thakker RV. Diagnosis of asymptomatic primary hyperparathyroidism: proceedings of the Fourth International Workshop. J Clin Endocrinol Metab 2014; 99: 3570-3579.

33. Martinez I, Saracho R, Montenegro J, Llach F. The importance of dietary calcium and phosphorous in the secondary hyperparathyroidism of patients with early renal failure. Am J Kidney Dis 1997; 29: 496-502.

34. Cusano N, Silverberg S, Bilezikian J. Chapter 22 - Normocalcemic PHPT. The Parathyroids (Third Edition). San Diego: Academic Press; 2015: 331-339.

35. Amaral LM, Queiroz DC, Marques TF, Mendes M, Bandeira F. Normocalcemic versus Hypercalcemic Primary Hyperparathyroidism: More Stone than Bone? J Osteoporos 2012; 2012: 128352.

36. Scholz DA, Purnell DC. Asymptomatic primary hyperparathyroidism. 10-year prospective study. Mayo Clin Proc 1981; 56: 473-478.

37. Fitzpatrick LA. Chapter 26 - Acute Primary Hyperparathyroidism A2 - Bilezikian, John P. The Parathyroids (Third Edition). San Diego: Academic Press; 2015: 401-408.

38. Cundy T, Darby AJ, Berry HE, Parsons V. Bone metabolism in acute parathyroid crisis. Clin Endocrinol (Oxf) 1985; 22: 787-793.

39. Caron P, Simonds WF, Maiza JC, Rubin M, Cantor T, Rousseau L, et al. Nontruncated amino-terminal parathyroid hormone overproduction in two patients with parathyroid carcinoma: a possible link to HRPT2 gene inactivation. Clin Endocrinol (Oxf) 2011; 74: 694-698.

40. Robertson WG, Marshall RW. Calcium measurements in serum and plasma--total and ionized. CRC Crit Rev Clin Lab Sci 1979; 11: 271-304.

41. Hossein-nezhad A, Holick MF. Vitamin D for health: a global perspective. Mayo Clin Proc 2013; 88: 720-755.

42. Tucci JR. Vitamin D therapy in patients with primary hyperparathyroidism and hypovitaminosis D. Eur J Endocrinol 2009; 161: 189-193.

43. Thakker RV. Familial and Hereditary Forms of Primary Hyperparathyroidism A2. En: Bilezikian JP, Marcus R, Levine MA, Marcocci C, Silverberg SJ, Potts JT, eds. The Parathyroids (ed 3a). California, Estados Unidos: Academic Press; 2015: 341-363.
44. Starker LF, Fonseca AL, Carling T, Udelsman R. Minimally invasive parathyroidectomy. Int J Endocrinol 2011; 2011: 206502.

45. Noussios G, Anagnostis P, Natsis K. Ectopic parathyroid glands and their anatomical, clinical and surgical implications. Exp Clin Endocrinol Diabetes 2012; 120: 604-610.

46. Kunstman JW, Kirsch JD, Mahajan A, Udelsman R. Clinical review: Parathyroid localization and implications for clinical management. J Clin Endocrinol Metab 2013; 98: 902-912.

47. Cheung K, Wang TS, Farrokhyar F, Roman SA, Sosa JA. A meta-analysis of preoperative localization techniques for patients with primary hyperparathyroidism. Ann Surg Oncol 2012; 19: 577-583.

48. Smith RB, Evasovich M, Girod DA, Jorgensen JB, Lydiatt WM, Pagedar NA, et al. Ultrasound for localization in primary hyperparathyroidism. Otolaryngol Head Neck Surg 2013; 149: 366-371.

49. Hetrakul N, Civelek AC, Stagg CA, Udelsman R. In vitro accumulation of technetium-99m-sestamibi in human parathyroid mitochondria. Surgery 2001; 130: 1011-1018.

50. Froberg AC, Valkema R, Bonjer HJ, Krenning EP. 99mTc-tetrofosmin or $99 \mathrm{mTc}$-sestamibi for double-phase parathyroid scintigraphy? Eur J Nucl Med Mol Imaging 2003; 30: 193-196.

51. Leslie WD, Dupont JO, Bybel B, Riese KT. Parathyroid $99 \mathrm{mTc}$-sestamibi scintigraphy: dual-tracer subtraction is superior to double-phase washout. Eur J Nucl Med Mol Imaging 2002; 29: 1566-1570.

52. Moralidis E. Radionuclide parathyroid imaging: a concise, updated review. Hell J Nucl Med 2013; 16: 125-133.

53. Wei WJ, Shen CT, Song HJ, Qiu ZL, Luo QY. Comparison of SPET/CT, SPET and planar imaging using $99 \mathrm{mTC}-\mathrm{MIBI}$ as independent techniques to support minimally invasive parathyroidectomy in primary hyperparathyroidism: A meta-analysis. Hell J Nucl Med 2015; 18: 127-135.

54. Kluijfhout WP, Vorselaars WM, Vriens MR, Borel Rinkes IH, Valk GD, de Keizer B. Enabling minimal invasive parathyroidectomy for patients with primary hyperparathyroidism using Tc-99m-sestamibi SPECT-CT, ultrasound and first results of (18)F-fluorocholine PET-CT. Eur J Radiol 2015; 84: 1745-1751.

55. Guerin C, Lowery A, Gabriel S, Castinetti F, Philippon M, Vaillant-Lombard J, et al. Preoperative imaging for focused parathyroidectomy: making a good strategy even better. Eur J Endocrinol 2015; 172: 519-526.

56. Berner AM, Haroon A, Nowosinska E, Offiah C, Luqman $M$, Newell $M$, et al. Localization of parathyroid disease with 'sequential multiphase and dual-tracer' technique and comparison with neck ultrasound. Nucl Med Commun 2015; 36: 45-52.

57. Starker LF, Mahajan A, Bjorklund P, Sze G, Udelsman R, Carling T. 4D parathyroid CT as the initial localization study for patients with de novo primary hyperparathyroidism. Ann Surg Oncol 2011; 18: 1723-1728. 
58. Mahajan A, Starker LF, Ghita M, Udelsman R, Brink JA, Carling T. Parathyroid four-dimensional computed tomography: evaluation of radiation dose exposure during preoperative localization of parathyroid tumors in primary hyperparathyroidism. World J Surg 2012; 36: 1335-1339.

59. Michel L, Dupont M, Rosiere A, Merlan V, Lacrosse $M$, Donckier JE. The rationale for performing MR imaging before surgery for primary hyperparathyroidism. Acta Chir Belg 2013; 113: 112-122.

60. Nael K, Hur J, Bauer A, Khan R, Sepahdari A, Inampudi $\mathbf{R}$, et al. Dynamic 4D MRI for Characterization of Parathyroid Adenomas: Multiparametric Analysis. AJNR Am J Neuroradiol 2015; 36: 2147-2152.

61. Michaud L, Balogova S, Burgess A, Ohnona J, Huchet V, Kerrou K, et al. A Pilot Comparison of 18F-fluorocholine PET/CT, Ultrasonography and 123I/99mTc-sestaMIBI Dual-Phase Dual-Isotope Scintigraphy in the Preoperative Localization of Hyperfunctioning Parathyroid Glands in Primary or Secondary Hyperparathyroidism: Influence of Thyroid Anomalies. Medicine (Baltimore) 2015; 94: e1701.

62. Kunstman JW, Udelsman R. Superiority of minimally invasive parathyroidectomy. Adv Surg 2012; 46: 171-189.

63. Naveh-Many T, Rahamimov R, Livni N, Silver J. Parathyroid cell proliferation in normal and chronic renal failure rats. The effects of calcium, phosphate, and vitamin D. J Clin Invest 1995; 96: 1786-1793.

64. Jorde R, Szumlas K, Haug E, Sundsfjord J. The effects of calcium supplementation to patients with primary hyperparathyroidism and a low calcium intake. Eur J Nutr 2002; 41: $258-263$

65. Marcocci C, Bollerslev J, Khan AA, Shoback DM. Medical management of primary hyperparathyroidism: proceedings of the fourth International Workshop on the Management of Asymptomatic Primary Hyperparathyroidism. J Clin Endocrinol Metab 2014; 99: 3607-3618.
66. Grey A, Lucas J, Horne A, Gamble G, Davidson JS, Reid IR. Vitamin $D$ repletion in patients with primary hyperparathyroidism and coexistent vitamin D insufficiency. J Clin Endocrinol Metab 2005; 90: 2122-2126.

67. Stewart ZA, Blackford A, Somervell H, Friedman K, Garrett-Mayer E, Dackiw AP, et al. 25-hydroxyvitamin D deficiency is a risk factor for symptoms of postoperative hypocalcemia and secondary hyperparathyroidism after minimally invasive parathyroidectomy. Surgery 2005; 138 : 1018-1025; discussion 1025-1016.

68. Khan AA, Bilezikian JP, Kung AW, Ahmed MM, Dubois $\mathrm{SJ}$, Ho AY, et al. Alendronate in primary hyperparathyroidism: a double-blind, randomized, placebo-controlled trial. J Clin Endocrinol Metab 2004; 89: 3319-3325.

69. Khan AA, Bilezikian JP, Kung A, Dubois SJ, Standish TI, Syed ZA. Alendronate therapy in men with primary hyperparathyroidism. Endocr Pract 2009; 15: 705-713.

70. Zanocco K, Angelos P, Sturgeon C. Cost-effectiveness analysis of parathyroidectomy for asymptomatic primary hyperparathyroidism. Surgery 2006; 140: 874-881; discussion 881-872.

71. Shoback DM, Bilezikian JP, Turner SA, McCary LC, Guo MD, Peacock M. The calcimimetic cinacalcet normalizes serum calcium in subjects with primary hyperparathyroidism. J Clin Endocrinol Metab 2003; 88: 5644-5649.

72. Peacock M, Bolognese MA, Borofsky M, Scumpia S, Sterling LR, Cheng S, et al. Cinacalcet treatment of primary hyperparathyroidism: biochemical and bone densitometric outcomes in a five-year study. J Clin Endocrinol Metab 2009; 94: 4860-4867.

73. Khan A, Bilezikian J, Bone H, Gurevich A, Lakatos P, Misiorowski W, et al. Cinacalcet normalizes serum calcium in a double-blind randomized, placebo-controlled study in patients with primary hyperparathyroidism with contraindications to surgery. Eur J Endocrinol 2015; 172: 527-535.

\begin{abstract}
Primary hyperparathyroidism is one of the most frequent endocrine disorders characterized by uncontrolled production of parathyroid hormone. This clinical condition could be a completely asymptomatic disorder or one with manifestations that threaten the patient life. The great variety in clinical manifestations and misinterpretation of the diagnostic test make the primary hyperparathyroidism a real challenge for physicians. An undiagnosed and in consequence an untreated primary hyperparathyroidism could carry detrimental consequences for patients including impaired of renal function, which may lead to renal replacement therapy, osteoporosis, which may be accompanied with fragility fractures, and neurocognitive changes, that can disturb the quality of life of the individual. For this reason, it is fundamental to know and understand hyperparathyroidism, as well as properly recognize and interpret the diagnostic tools to be capable to propose an adequate treatment plan that fortunately in most cases leads to the cure of this disorder.
\end{abstract}

Key words: Primary hyperparathyroidism, parathyroid hormone, hypercalcemia. 


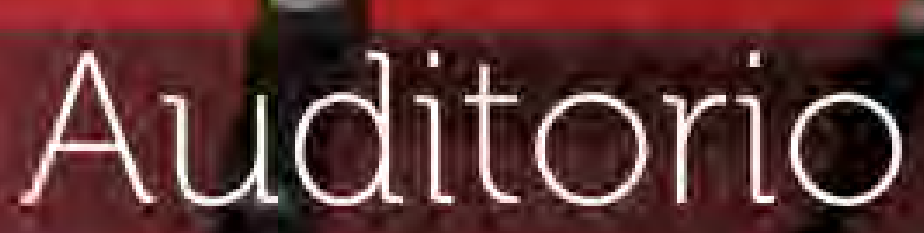

Un espacio a su servicio

Auditorio tipo teato

Ideal para convenciones, reuniones empresariales, conferencias, seminanen.

cursos, foros y muestras comerciales

Capacidad para 150 personas

Sistema de sonido acustico profesiend

Parqueadero robotizado para 95 vehiculos

Ubicado en el moderno edificio del Laboratorio Clínico Hematológico

Carrera 43 C \# 5 - 33. Patio Bonito, El Poblado Medellin - Antioquia

Mayor información: 4444900

EDIMECO

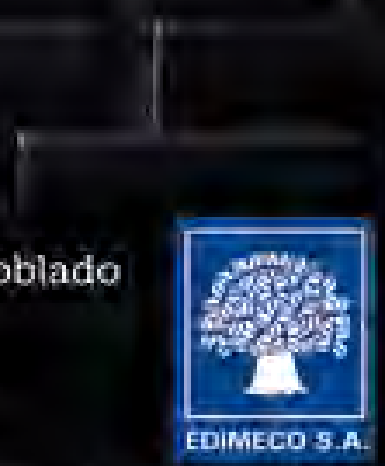

